\title{
Multivariate Analysis and Compressed Sensing Methods for Spectroscopic Electron Tomography of Semiconductor Devices
}

Martin Jacob ${ }^{1}$, Toby Sanders ${ }^{2}$, Nicolas Bernier ${ }^{1}$, Adeline Grenier ${ }^{1}$, Rafael Bortolin Pinheiro ${ }^{1}$, Frédéric Mazen $^{1}$, Pascale Bayle-Guillemaud ${ }^{3}$, Zineb Saghi $^{1}$

1. Univ. Grenoble Alpes, CEA, LETI, Grenoble, France

2. School of Mathematical and Statistical Sciences, Arizona State University, Tempe, AZ, USA

3. Univ. Grenoble Alpes, CEA, INAC, Grenoble, France

Spectroscopic electron tomography (ET) has recently gained momentum thanks to the advances in instrumentation in electron energy loss spectroscopy (EELS) and energy-dispersive X-ray spectroscopy (EDX) [1]. It however requires long exposure times and high beam currents compared to conventional ET, and results in huge multidimensional sets of data even when selecting relatively large tilt increments. It is therefore necessary to use sophisticated machine learning and tomography algorithms for spectral dimensionality reduction and $3 \mathrm{D}$ reconstruction.

In this work, we applied different multivariate statistical analysis strategies to a STEM-EELS tomographic dataset, and illustrated the strengths and weaknesses of total variation minimization (TVM) [2] algorithm for the $3 \mathrm{D}$ volume reconstruction.

A silicon fin-shaped structure implanted with arsenic (As) as n-type dopant and encapsulated in silicon oxide $\left(\mathrm{SiO}_{2}\right)$, was chosen as a test object. A needle-shaped sample was prepared by focused ion beam (FIB) and transferred onto an on-axis tomography holder. The experiment was performed at $200 \mathrm{kV}$ using a double-corrected FEI microscope equipped with a Gatan Quantum energy filter. 23 STEM-EELS datacubes, covering a tilt range of $180^{\circ}$, were acquired using Gatan Microscopy Suite software. A frame size of 60x90 pixels was selected, with a pixel size of $1 \mathrm{~nm}$ and an acquisition time of $0.05 \mathrm{sec} /$ pixel for the energy range of $300-1324 \mathrm{eV}$ and $0.5 \mathrm{sec} / \mathrm{pixel}$ for the energy range of $1100-2124 \mathrm{eV}$. Spectral unmixing using non-negative matrix factorization (NMF) [3] and spectral vertex components analysis (VCA) [4] was performed to decompose the data into a given number of spectral components and abundance maps following a linear mixing model. Both VCA and NMF ensured a physical meaning of the extracted spectral components by imposing a non-negativity constraint in NMF and assuming endmembers are present among the observed pixels in VCA. In Figure 1, we show the results obtained with VCA, after subsampling in energy to allow a reasonable computing time: 4 spectral components were extracted from the core-loss spectra, corresponding to $\mathrm{Si}(\mathrm{b}), \mathrm{As}(\mathrm{c}), \mathrm{SiO}_{2}$ (d) chemical phases, and the vacuum signal (c) which was used to mask the input images (similar decomposition was achieved with NMF). The tilt series of the $\mathrm{Si}, \mathrm{SiO}_{2}$ and As abundance maps were aligned in ImageJ and reconstructed using TVM, and the more conventional simultaneous iterative reconstruction technique (SIRT) [5] for comparison. Figure 2 shows the volume rendering of the fin obtained with SIRT (a) and TVM (b), and selected y-z (c,d) and x$\mathrm{z}(\mathrm{e}, \mathrm{f})$ cross-sections through the reconstructions. The piece-wise constant constraint imposed in TVM led to a noise reduction for the three phases and a better quality reconstruction of the As precipitates, for which sparsity in the gradient domain is justified. TVM is however not optimized for analyzing chemical diffusion and implantation. Important parameters such as 3D-dopant distribution inside the fin structure, 
lateral profile and sidewall dose could be erroneously overestimated with TVM (Figure 2(d)). To overcome these limitations, alternative sparsifying transforms such as wavelets and curvelets will be tested and benefits/drawbacks of each method will be discussed.

\section{References:}

[1] SM Collins and PA Midgley, Ultramicroscopy 180 (2017), p. 133.

[2] C Li et al, Computational Optimization and Applications, 56 (2013), p. 507.

[3] M Shiga et al, Ultramicroscopy 170 (2016), p. 43.

[4] JMP Nascimento and JM Bioucas-Dias, IEEE Transactions on geoscience and remote sensing, 43 (2005), p. 898.

[5] PFC Gilbert, Journal of Theoretical Biology 36 (1972), p. 105.
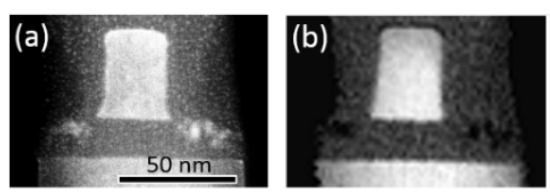

(d)
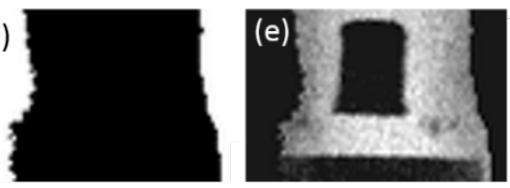
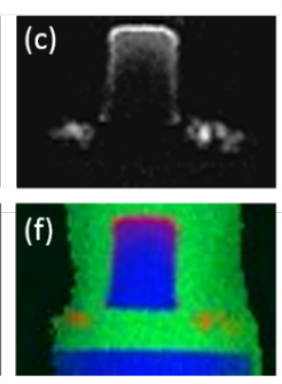

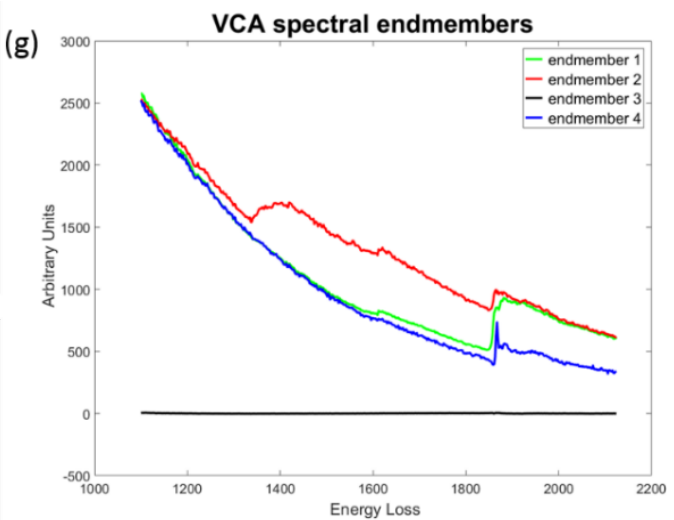

Figure 1. STEM-EELS tomography of a FinFET. (a) STEM-HAADF image, (b-e) VCA abundance maps (corresponding to $\mathrm{Si}, \mathrm{As}$, mask and $\mathrm{SiO}_{2}$ ) and the corresponding spectral components (g) extracted from the entire STEM-EELS tomographic dataset. (f) shows a superposition of $\mathrm{Si}$ (blue), $\mathrm{SiO}_{2}$ (green) and As (red) maps.
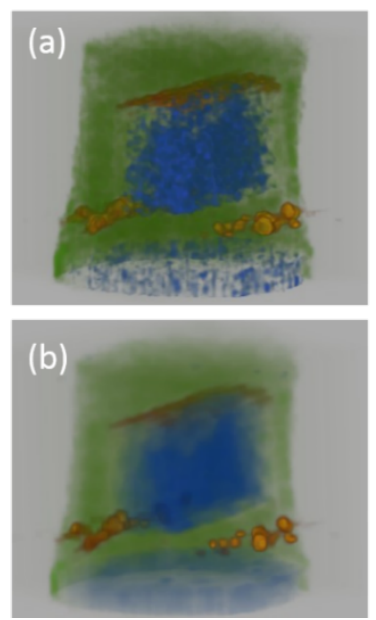
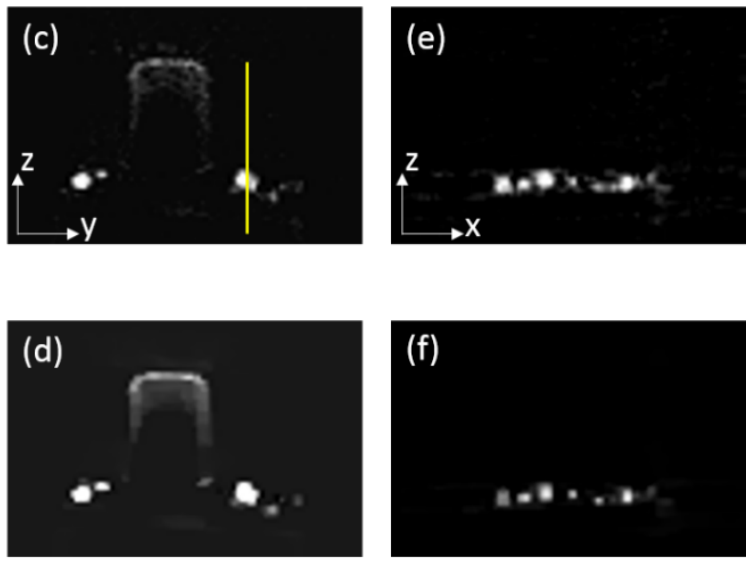

Figure 2. Voxel rendering of the SIRT (a) and TVM (b) reconstructions of the FinFET ( $\mathrm{Si}$ (blue), $\mathrm{SiO}_{2}$ (green) and As (orange)); y-z slice through the SIRT (c) and TVM (d) reconstruction of the As chemical volume; $x-z$ slice through the SIRT (e) and TVM (f) reconstruction of the As chemical volume at the $y$ position corresponding to the yellow line in (c). 